\title{
Fast harmonic analysis for PHIL experiments with decentralized real-time controllers
}

\author{
Erhan Sezgin*, Anurag Mohapatra ${ }^{\dagger}$, Vedran S. Perić ${ }^{\dagger}$, Özgül Salor ${ }^{\S}$, Thomas Hamacher $^{\dagger}$ \\ ${ }^{*}$ Department of Electrical and Electronics Engineering, Kafkas University, Kars, Turkey \\ ${ }^{\dagger}$ CoSES, Munich School of Engineering, Technical University of Munich, Munich, Germany

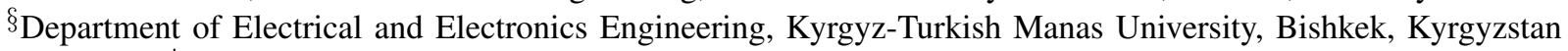 \\ $\ddagger$ Department of Electrical and Electronics Engineering, Gazi University, Ankara, Turkey
}

\begin{abstract}
This paper proposes and implements, a harmonic analysis algorithm for microgrid Power Hardware-in-the-loop (PHIL) experiments, when the point of common coupling (PCC) voltage cannot be directly wired to the local prosumer controllers due to long distances between them. Using frequency-shifting and filtering ideas, the voltage measurement is converted to magnitude and phase information. This is passed over an asynchronous communication link to another controller, where it is recovered into a waveform after delay compensation. The method allows for accurate power calculations and grid synchronization over distributed prosumer controllers. The proposed method can work at different execution rates depending on real time (RT) workload and is shown to be robust against step changes, harmonics and communication delays. The method is demonstrated with two PHIL experiments at the CoSES, TU Munich lab in grid connected and island mode.
\end{abstract}

Index Terms-Power Hardware-in-the-loop, distributed control, prosumers, frequency shifting, DFT.

\section{INTRODUCTION}

Fully controllable and dispatchable, power electronics based distributed energy resources (DERs) have shown a great potential for more efficient and reliable grid operation. Combined with the advent of Information and Communication Technology (ICT) for power systems and flexibility offered through sector coupling, the literature on central and local control of prosumer oriented, multi energy systems has grown rapidly. Accurate and fast measurements within a prosumer microgrid are an important domain of research in multi energy systems. Within this broad topic, the location of the measurements with respect to the location of the controller is of particular interest.

An increase in individually controlled prosumers can lead to situations where the the relevant point of common coupling (PCC) voltage might not be available on-site. The PCC voltage is important to accurately calculate power injection from the prosumers and it is generally taken at the distribution grid node behind the PCC. In the context of an optimized microgrid, each prosumer contributes individually and collectively towards maintaining the grid node voltage within limits. However, multiple prosumers can be physically too far removed to send direct measurements of the PCC voltage, as seen in a schematic in Fig. 1 .

The Center for Combined Smart Energy Systems (CoSES) at TU Munich was established to research sector coupled, low-inertia, active distribution grids [1]. It emulates multiple

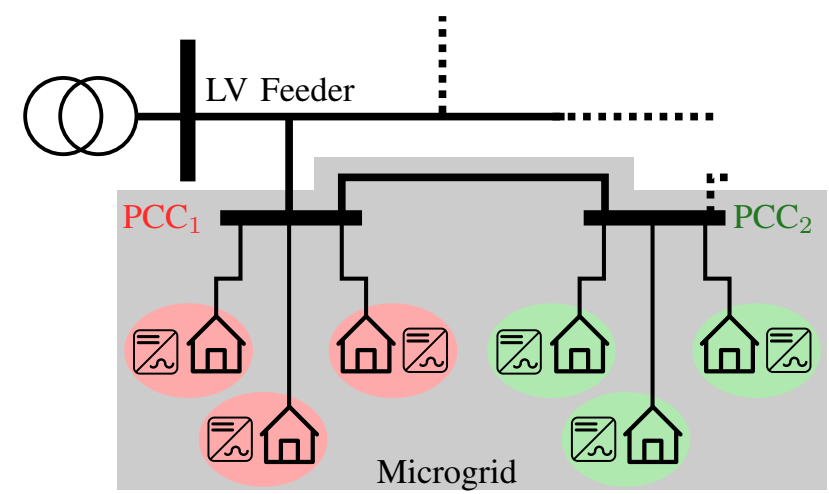

Fig. 1. Microgrids at Low Voltage (LV) level with individually controlled prosumers (the control area of each prosumer is seen in and can be located too far away from PCC for direct wiring of voltage measurements.

fully controlled electrical and heat prosumers in a local grid, based on Power Hardware-in-the-Loop (PHIL) philosophy, to represent a real world multi energy grid and eliminates the need for real-time (RT) simulators. In CoSES, the grid voltage measurements are acquired at a controller separate from the prosumer controllers. Each prosumer has its own embedded controller which decides the local power injection. While the current is measured locally at the prosumer bus, the voltage of the upstream distribution grid node is missing. The instrumentation cabling length between the location of the voltage measurements and the prosumer controllers makes it impossible to send these measurements directly.

Therefore, continuous voltage measurements required as feedback for prosumer injection control must be sent over an asynchronous communication link. To avoid loss of information, it is prudent to perform harmonic analysis on the voltage and send the magnitude and phase values over the communication channels to local prosumer controllers. The harmonic analysis models must also be accurate and show quick convergence. Furthermore, the control philosophy in CoSES requires the controllers to carry compiled RT models for prosumer emulation, energy management systems and DER control. Hence the signal processing must be kept easy to implement to allow capacities for the RT operation of the lab.

In this paper we present the development and implementation of a harmonic analysis and power measurement unit within the aforementioned constraints of the CoSES lab. 
To the best knowledge of the authors, there has been no previous work on voltage measurements collected at different controllers to calculate a RT feedback for PHIL control. We use a method involving frequency-shifting and filtering to continuously calculate the magnitude and phase components of the measured signal. We time-stamp the signal at the sending end controller and compensate for the communication delay on the receiving end of the prosumer controller. The prosumers, which are emulated over bi-directional inverters, require sample-by-sample setpoints of the reference current for a specific power injection. We recover the voltages as waveforms, using the transferred magnitude and phase information, and calculate the continuous current setpoints for the prosumer. We test our algorithm for accuracy through step changes and additional harmonics on the grid voltage while maintaining constant power injection from a prosumer.

The remainder of this paper is organised as follows. In Sec. II. we introduce the proposed methodology for the measurements and power calculation. Relevant literature on similar approaches is presented and analysed for their suitability for our use case. In Sec. III] we explain the electrical prosumers and the RT control structure for the decentralized controllers in our lab. In Sec. IV] we validate our methodology and then use it for two PHIL experiments to demonstrate its usefulness in the lab.

\section{Methodology}

The IEEE standard defines, power as the continuous multiplication of voltage and current waveforms [2]. Active power is further defined as the integration of this multiplication, averaged over certain whole number multiples of the fundamental frequency. In this paper we are concerned with the active power associated with the fundamental frequency which is also known as positive sequence power in some literature. In a practical sense, the positive sequence power is the useful power used in electrical appliances, devices and components used for power delivery. Therefore, our proposed method should primarily estimate the fundamental frequency in measurements and if needed other harmonic orders for specific PHIL experiments.

Stationary AC electrical signals can be expressed using line spectra model, where signals have constant frequencies with magnitudes and phase angles for each frequency. Discretizing the time with a sampling frequency $f_{s}$, an AC signal is obtained as given in (1) where $f$ is fundamental frequency. Here $x[n]$ has harmonic components with orders of $m(m \in[1, l])$, amplitudes $A_{m}$ and phase shifts $\phi_{m}$.

$$
x[n]=\sum_{m=1}^{l} A_{m} \cos \left(\frac{2 \pi m f n}{f_{s}}+\phi_{m}\right)
$$

The literature on harmonic analysis in general is well established. These are generally, Discrete Fourier Transform (DFT), Fast Fourier Transform (FFT) [3], Discrete Wavelet Transform (DWT) [4], Second Order Generalized Integrator (SOGI) [5] and Kalman Filter (KF) [6] based methods. However, their suitability for RT experiments for control feedback must be assessed. For our application of PHIL control in microgrids, we are looking for an method which is feasible in RT, is robust against step changes or harmonics and can preferably work with continuous signals.

DFT based methods are common in Power quality (PQ) applications. However FFT analysis is computationally superior over DFT and feasible in RT applications. Yet both methods still calculate all harmonics limited by sampling frequency and thus are computationally expensive for online applications. Buffering the signals to reduce burden is not suitable as this increases the convergence time of the estimates. Another issue is the change of signal and time localisation of the estimates within large buffers. DWT based methods promise to be suitable for these changes but again the computation burden is drastically increased for RT applications [4]. KF based methods are robust against non-stationary signals [6], but the computation burden makes it infeasible for RT applications. SOGI based methods have the required computation efficiency for RT use and do not buffer the signal [5]. However, they do not outperform FFT type algorithms in convergence after step changes. KF and SOGI type methods also need a priori information on harmonic content and thus perform poorly in presence of unexpected harmonics.

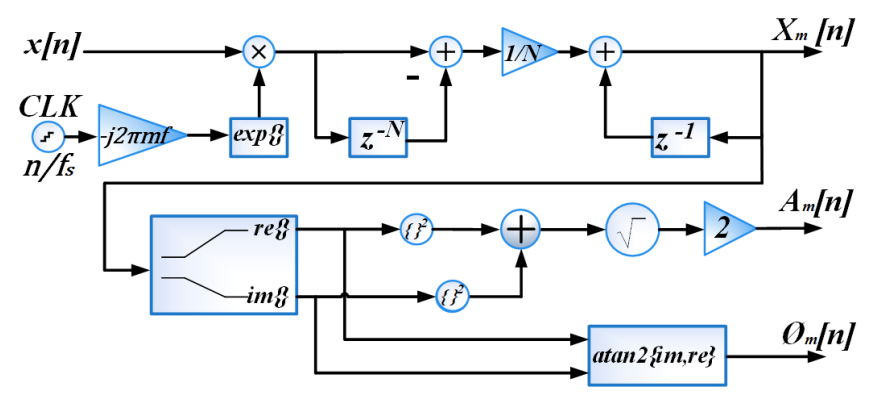

Fig. 2. Proposed harmonic analysis method

\section{A. Proposed Method}

We propose an approach where the measurements are subjected to amplitude modulation with synthetic complex exponential references followed by filtering. A block diagram representation of this proposed method is shown in Fig. 2. This concept is also known as, frequency-shifting and filtering [7], Coulon Oscillator [8], Quadrature Amplitude Modulation [9] or Complex Exponential Modulation[10], [11]. They all involve shifting the required frequency component to $0 \mathrm{~Hz}$ for best results on the filtering step that follows. The complex exponential reference can be produced continously from the RT controller clock signal. This gives us the benefit to timestamp without time localisation issues, transfer and recover the estimated signals in other controllers. A Moving Average (MA) filter generates a single coefficient in each step of the calculation. These coefficients hold the magnitude and phase information of the frequency component we wanted to estimate, as shown in 2. 


$$
X_{m}[n]=\frac{1}{N} \sum_{i=n-N+1}^{n} x[i] e^{\frac{-j 2 \pi m f i}{f_{s}}}
$$

In (2) the samples of $x[n]$ is multiplied with the corresponding element of the synthetic complex exponential reference along time and $N$ point MA filter is used to extract the parameters of $m^{\text {th }}$ harmonic component. $N$ stands for the fundamental period of the $x[n]$ signal and it is directly affected by the components of the signals itself.

The proposed algorithm can be used on fundamental and any harmonic component of the signal. But $N$ can also be chosen as multiples of the fundamental period to gain better frequency resolution. Such an averaging operation in (2) requires summation over $N$ points. This is a recursive calculation, where the average value is changed by en-queuing and de-queuing elements. To reduce the number of sum operators on this function, the average values can be calculated by using consecutive elements of modulated signal. To make this possible a former coefficient obtained from the signal is written as given in (3).

$$
X_{m}[n-1]=\frac{1}{N} \sum_{i=n-N}^{n-1} x[i] e^{\frac{-j 2 \pi m f i}{f_{s}}}
$$

Subtracting (3) from (2), gives us (4) which gives us the coefficients for each harmonic recursively. This averaging concept is known in literature as Cascaded Integrator-Comb (CIC) Moving Average Filter [12].

$$
\begin{aligned}
X_{m}[n]= & X_{m}[n-1]+ \\
& \frac{1}{N}\left(x[n] e^{\frac{-j 2 \pi m f n}{f_{s}}}-x[n-N] e^{\frac{-j 2 \pi m f(n-N)}{f_{s}}}\right)
\end{aligned}
$$

Using the coefficients calculated by (4), magnitude and phase angles of the harmonic components can be calculated as given in $5 \mathrm{a}$ and $5 \mathrm{~b}$ ) respectively.

$$
\begin{aligned}
A_{m} & =2 \times \sqrt{\Re\left(X_{m}[n]\right)^{2}+\Im\left(X_{m}[n]\right)^{2}} \\
\phi_{m} & =\arctan 2\left(\Im\left(X_{m}[n]\right), \Re\left(X_{m}[n]\right)\right)
\end{aligned}
$$

Waveforms for $x_{m}[n]$ can be calculated by combining real and imaginary parts of the coefficients with corresponding complex terms of the modulation signal as shown in (6a), or as in (6b), where the calculated magnitude and phase angles are combined with the instantaneous time and a sinusoidal function. The approach of $(6 \mathrm{~b})$, proves to be better in a distributed computation environment. A block diagram representation of the two methods is shown in Fig. 3

$$
\begin{gathered}
x_{m}[n]=\Re\left(e^{\frac{-j 2 \pi m f n}{f_{s}}}\right) \times \Re\left(X_{m}[n]\right)+ \\
\Im\left(e^{\frac{-j 2 \pi m f n}{f_{s}}}\right) \times \Im\left(X_{m}[n]\right) \\
x_{m}[n]=A_{m} \cos \left(\frac{2 \pi m f n}{f_{s}}+\phi_{m}\right)
\end{gathered}
$$
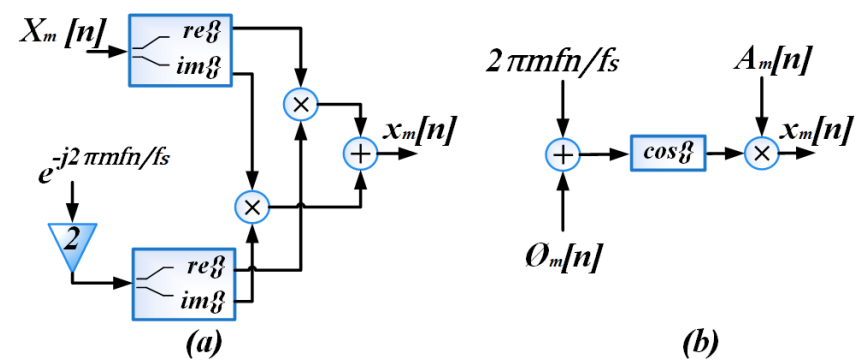

(b)

Fig. 3. Methods to generate waveforms from results of (4) and (5.

This proposed algorithm is similar to calculating equivalent coefficients with Modulated Sliding Discrete Fourier Transform (mSDFT) [13] under certain circumstances. mSDFT is a stable and computationally efficient algorithm which has been used in other RT applications [14], [15].

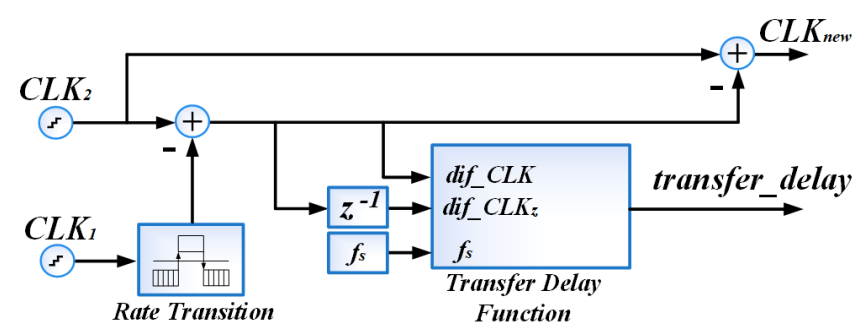

Fig. 4. Compensation of transfer delay to aid recovery of signals

Processing rate and time synchronization: Typically, decomposed signals are reconstructed at the same sampling frequency. The RT controllers in CoSES can be used for a variety of control and regulation tasks. It is therefore preferable to have models which can operate at different decimation of the target rate to manage RT workload. Both the harmonic estimation and recovery models as proposed in this paper can be run at independent rates. Thus the harmonic estimation model can run at a low rate to accommodate the burden of one model for each of the LV buses. On the recovery side, the models can run at a higher rate to generate precise setpoints for prosumer emulators.

Time synchronization between multiple RT controllers is required for our approach to make sure the timestamps on sending and receiving end of the voltages are comparable. However in distributed PHIL applications, we cannot necessarily ensure that models start execution across two controllers at the same time, even though the clocks are synchronized. In other words, two model clocks can have the same rate of change but show different time. Therefore a transfer delay function is introduced, explained in Algorithm. 1. which takes the sending and receiving end timestamp as input. It produces a new clock signal for the recovery model and calculates the correct transfer delay between the two ends. A block diagram for the whole delay compensation block is shown in Fig. 4

\section{B. Contribution}

In summary, the contribution of from our proposed method can be listed as follows- 


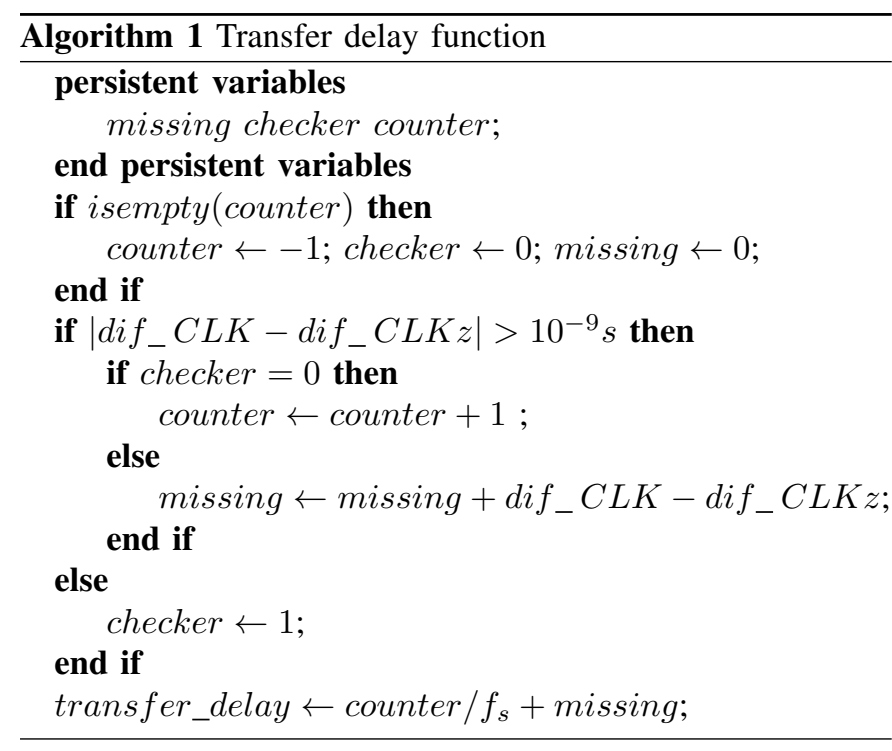

- Since actual timestamp of the independent computation device is included in the calculations, it is easy to synchronize the measurements processed on independent devices in a decentralized/distributed environment.

- Decomposition and reconstruction models can be run on independent controllers using the local timestamps.

- Decomposition and reconstruction models can be run with different data processing rates to use RT resource effectively and gain higher precision on waveforms if permitted.

- Compared to SDFT and recursive DFT calculations, the proposed method produces phase shifts $\left(\phi_{m}\right)$ on each sample rather than $\left(\frac{2 \pi m f n}{f_{s}}+\phi_{m}\right) \sqrt{5 \mathrm{~b}}$. This removes the need for usage of further reference signals to get the actual phase shift at each computation step.

\section{LABORATORY SETUP}

CoSES is structured as a small microgrid with coupled electrical and heat sectors for five prosumers. The electrical part of the lab is of particular interest for this paper and is shown as a schematic in Fig. 5. On the left side, we see the CoSES LV grid, made of $70 \mathrm{~mm}^{2}$ and $95 \mathrm{~mm}^{2}$ threephase four-core power cables sections, with a total length of approximately $2 \mathrm{~km}$. The length of cable and connection pattern between the ten LV buses can be changed to fit any specific grid structure for an experiment. Two tap changing transformers connect the lab to the Munich LV grid. Two extra circuit breakers are also provided to couple the transformer buses and to create a meshed grid, respectively [1].

Prosumer emulators: Each of the five prosumers bus has access to local DERs and a bi-directional inverter, which doubles as the prosumer emulator. These are part of the Egston COMPISO System Unit (CSU) [16], which acts as the PHIL component for CoSES. The Egston CSU at CoSES consists of seven 4-leg inverter cabinets which share the same DC bus. They serve as programmable bi-directional energy flow devices to emulate prosumer behaviour. The Egston CSU can

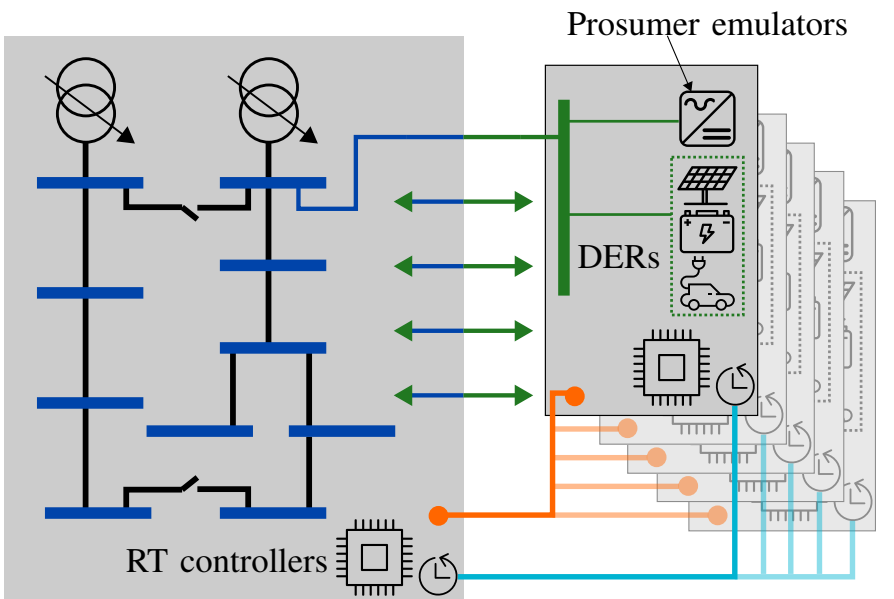
Grid control area
Access to all bus voltages and
current

Fig. 5. CoSES electrical grid with the five prosumers. Prosumer buses can be flexibly connected to any LV grid bus $\ldots$. An asynchronous communication link connects different control areas within the lab. The controller clocks are synchronized through a 1588 PTP ethernet link or through GPS.

inject currents up to $5 \mathrm{kHz}$ and thus can also be used as an active power filter in the grid.

Controllers: CoSES uses National Instruments (NI) hardware, PXIe-8880, and software, NI Veristand [17], for RT control systems of the electrical grid. Each of the five prosumers have a local RT controller and the sixth controller is located inside LV grid switchroom. The controllers act as a general purpose RT computer and has embedded systems capabilities through additional IO cards. They can also send injection setpoints to the Egston CSU through a SFP link. Within CoSES, they operate at either $5 \mathrm{kHz}$ or $10 \mathrm{kHz}$ RT target rate during HIL experiments. An asynchronous communication ring, shown in orange in Fig. 5, called Reflective Memory Network (RMN) is used to make data recorded or computed in any distributed controller available at any other controllers under a $1 \mathrm{~ms}$ jitter. The software environment, NI Veristand accepts compiled models from tools such as MATLAB/Simulink, LabVIEW, SimulationX and Python. The environment can run models at different execution rates, access field measurements and map IOs between models and hardware.

Time Synchronization between RT controllers: Synchronization is an integral part of any HIL test bench with distributed control. However, as explained in Sec. III, it attains a higher significance in CoSES due to the voltage and current measurements for a prosumer being split over at least two controllers, one local and the other in the LV Grid switchroom. RT Controllers in CoSES are synchronized through the NI$6683 \mathrm{H}$ timing and sync card, which slots into the PXIe chassis. The card provides synchronization over the 1588 Precise Time Protocol (PTP) or through a GPS antenna, as seen in cyan in Fig. 5. The HIL model clocks are thus synchronized to around $5 \mu \mathrm{s}$, as measured over experiments. A further synchronization is required for the PHIL emulator, Egston CSU, as it has a separate FPGA controller per cabinet. This is achieved over 


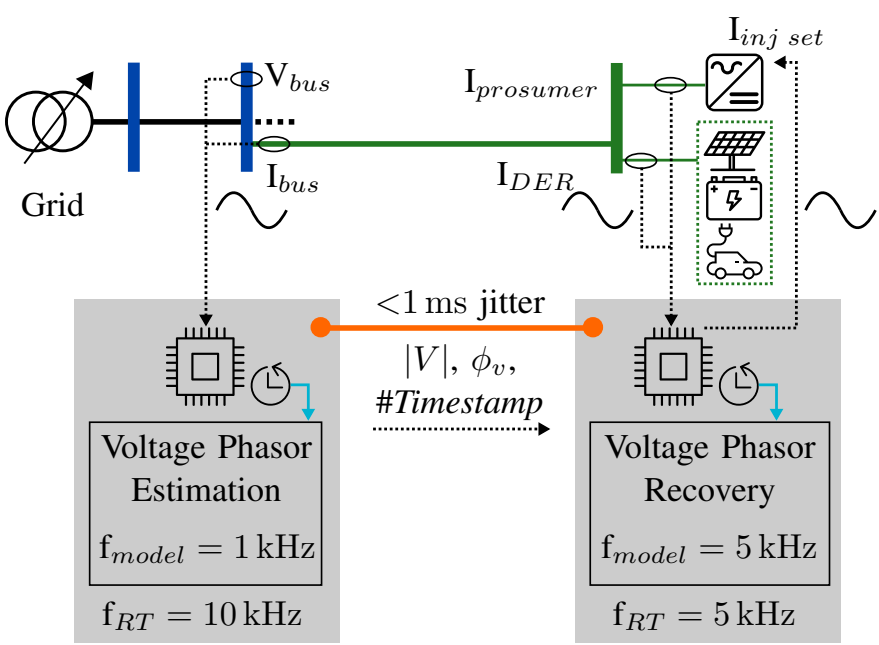

Fig. 6. Schematic representation of the grid synchronization and injection power measurement concept in CoSES.

the SFP link, through a $4 \mu$ s strobe signal, which makes the Egston CSU clock a slave to the RT controller clocks. Thus, a laboratory wide time synchronization is achieved for measurements, PHIL emulators and RT controllers.

Sensors: A total of 246 current and voltage measurements are made in the CoSES electrical grid through LEM transducers [18]. These measurements are wired to the nearest relevant RT controller and are acquired at the controller target rate. As shown in Fig. 5, the grid control area has all the LV bus measurements and these are wired to the RT grid controller. At the prosumer level, the local currents from Egston CSU and DERs are wired to the local RT prosumer controller.

\section{EXPERIMENTAL VALIDATION AND ANALYSIS}

A schematic of the distributed measurements being used for grid synchronization and injection power calculation is shown in Fig. 6. The voltage of the LV bus (PCC) is measured at the grid controller, shown on the left. This analog measurement is fed to a phasor estimation model running at a specific execution rate. The output of this model, $|V|$ and $\phi_{v}$, is sent out over the orange asynchronous link to the downstream prosumer's local controller, as seen in Fig. 6 on the right. These outputs are also timestamped at the grid controller. The prosumer controller, which is given a power setpoint by the operator, uses the transferred data as inputs for phasor recovery model, running at a specific execution rate. Since the controller clocks are synchronized, this model corrects for the communication delay by comparing the incoming timestamp from the grid controller to its current controller time using the idea from Fig. 4 The model then outputs the current setpoint waveform to be fed sample-by-sample to the Egston CSU. The injected power of the prosumer as a whole can be measured at the grid controller or individually from the Egston CSU and DERs at the local prosumer controller.

\section{A. Validation of phasor estimation and recovery method}

We first validate the phasor estimation and recovery models, shown in Fig. 6, with measurements in an island mode with
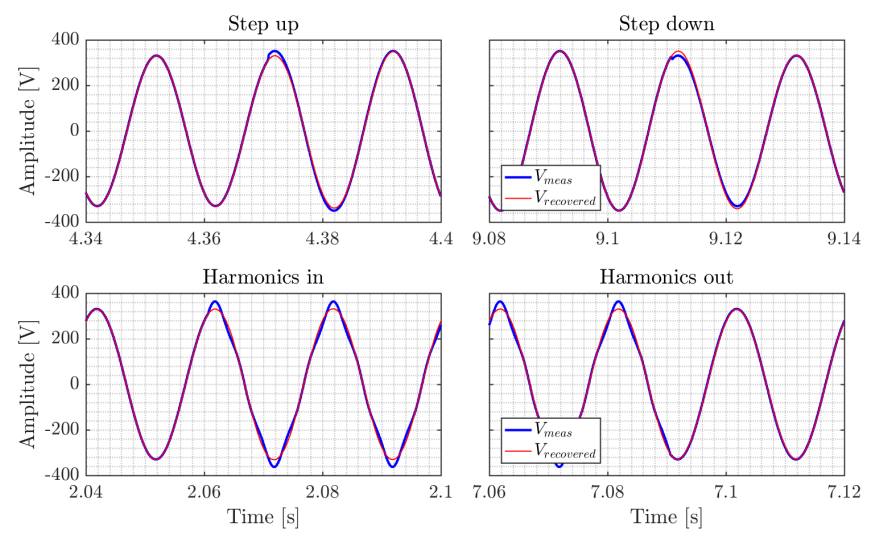

Fig. 7. Validation of recovered waveform under step and harmonic changes

a controlled voltage source. The models use the algorithm as mentioned in Sec. II-A. The island grid voltage from one LV bus, is measured at the RT grid controller and then analysed at $1 \mathrm{kHz}$ to estimate the $|V|$ and $\phi_{v}$. This is transfered to a prosumer controller, which recovers the voltage, at $5 \mathrm{kHz}$, as a waveform after delay compensation. Both the measured and the recovered voltages are logged individually in the two separate controllers. The results are plotted in Fig. 7 with the $\mathrm{x}$-axis values being the local timestamp from the controllers at the point of logging.

In Fig. 7, we see the models reacting to a step change in the voltage amplitude from $330 \mathrm{~V}$ to $350 \mathrm{~V}$ and back. It can be seen that convergence between the recovered and measured waveform is achieved in approximately one cycle. In Fig. 7, $3^{\text {rd }}$ and $5^{\text {th }}$ harmonics are added to the island grid voltage. The harmonic magnitude is taken at $5 \%$ of the fundamental amplitude. The recovered voltage is unaffected as it is able to distinguish the fundamental component of the distorted measured signal. The results shows accuracy, quick convergence and robustness of the estimation and recovery models against changes in the measured voltage.

\section{B. Implementation as a tool in PHIL experiments}

Two PHIL experiments are conducted using the distributed measurement concept validated in the previous section. In Fig. 8. we use Prosumer\#1 to act as a grid forming inverter with a $V_{\text {grid ref }}$ given through the operator GUI. The same GUI also sends an active power setpoint $P_{2}$ set to Prosumer\#2 which acts as a grid following inverter. The injected power from Prosumer\#2 is also sent back to the GUI for logging. The grid voltage is analysed in the Grid controller and sent to the Prosumer\#2 controller to calculate the correct $I_{i n j}$ set for constant $P_{2}$ measured regardless of the change in $\hat{V}_{\text {grid ref }}$. As the Egston CSUs always consume the some amount of reactive power based on the active power reference, the injected active power does not exactly coincide with the reference in most cases. Therefore a PI-based controller is used to modify the setpoint slightly to achieve exact injected active and reactive power. As this power control loop affects the convergence rate of the measurements, we show two sets of result, one with and one without the power controller. 


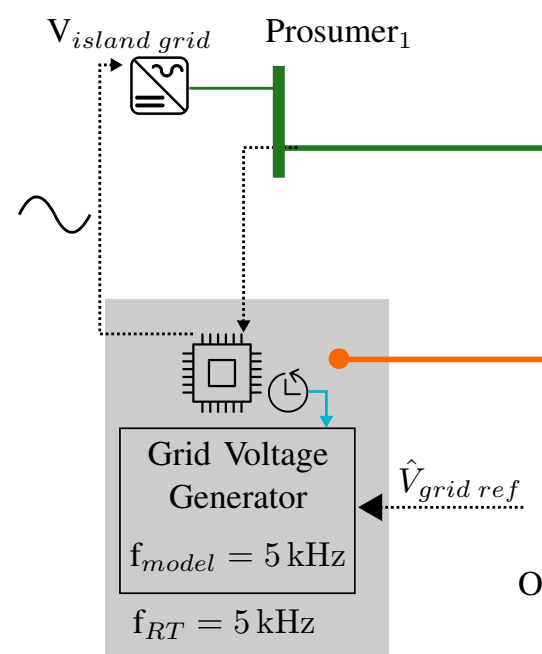

Prosumer controller \#1

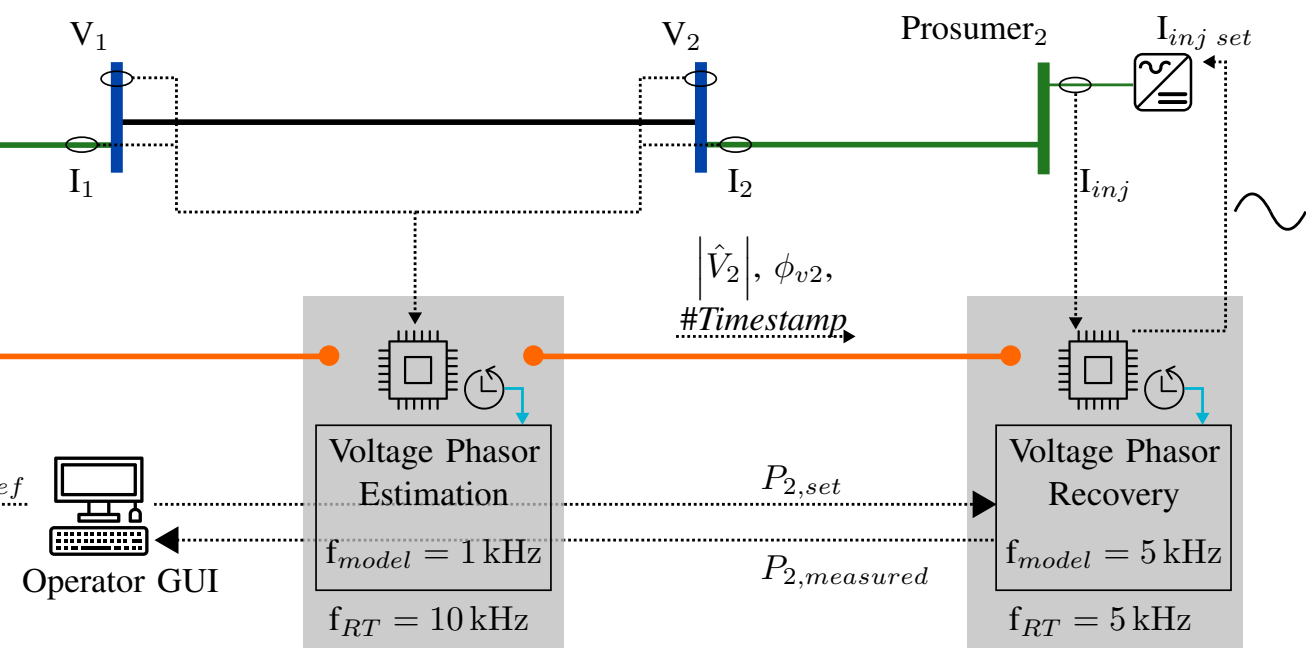

Grid controller

Prosumer controller \#2

Fig. 8. Schematic of the PHIL experiment for constant power injection under step change or distortion of grid voltage in islanding mode. Prosumer\#1 acts as the grid forming inverter and Prosumer\#2 as grid following inverter with a fixed active power setpoint. Grid controller measures and analyses the voltages.
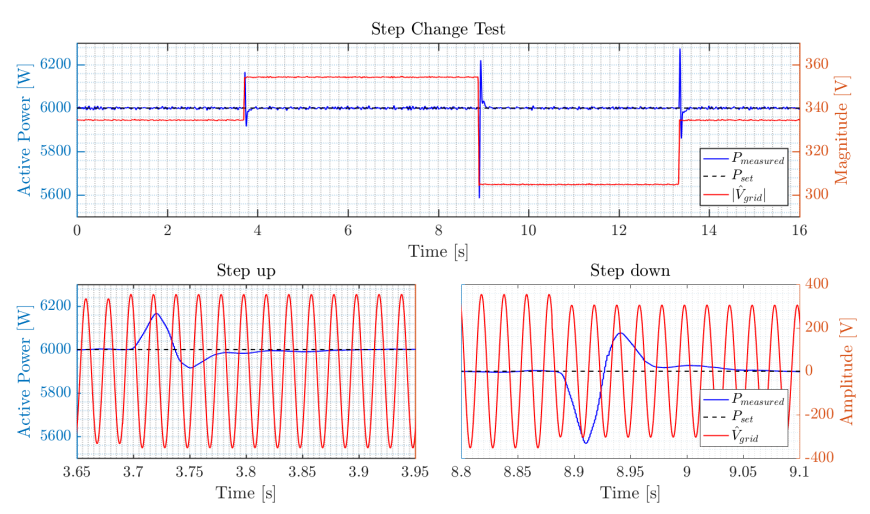

Fig. 9. Step change test on voltage with power control loop
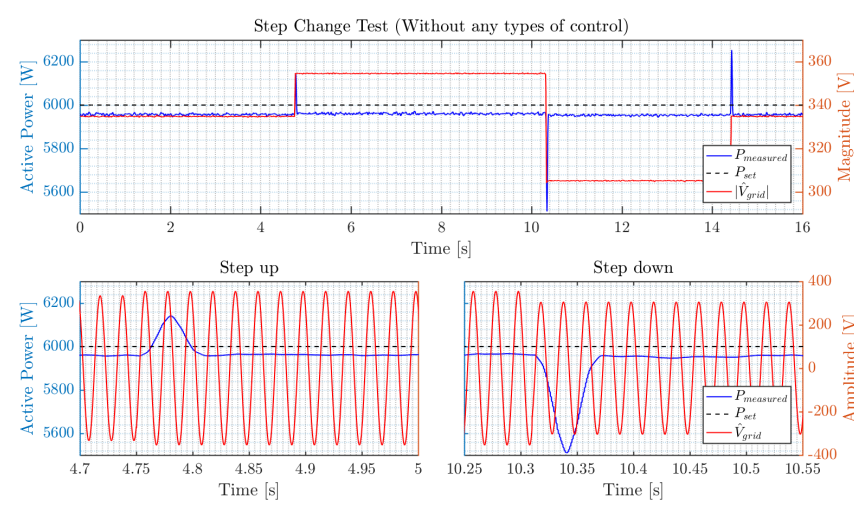

Fig. 10. Step change test on voltage without power control loop

In Fig. 9 and Fig. 10 we see the results as $\hat{V}_{\text {grid ref }}$ is changed from $330 \mathrm{~V}$ to $350 \mathrm{~V}$, then to $300 \mathrm{~V}$, and back to $330 \mathrm{~V}$ with a constant $P_{2}$ set $=6 \mathrm{~kW}$. In Fig. 9, we see that the $P_{2}$ measured converges to $P_{2}$ set in around 6 cycles of the fundamental. We then turn-off the power controller and repeat the measurements in Fig. 10. Now the $P_{2}$ set and $P_{2}$ measured have a constant steady state error. However, the power measurement converges inside 2 cycles of the fundamental. These results are consistent with the convergence rate of Fig. 7, as the extra cycle is being used by the moving average part of the power measurement block.

We conduct a further PHIL experiment to minimize net grid export due to excess PV generation and the schematic is shown in Fig. 11. The LV buses are now connected to the Munich grid and thus the controlled prosumers must be synchronized using the information from the measurement models. The grid controller, as usual sends the measured voltages, $V_{1}$ and $V_{2}$, of the two LV buses to their respective downstream prosumer controllers. It additionally measures the exported power to the upstream Munich grid. Prosumer\#1 measures the local DER power from the PV. This power is sent as $P_{2}$ set to Prosumer\#2, which emulates a dynamic load to consume the total PV power. The load is measured as $P_{2}$ measured and sent to the operator GUI. We being the experiment with Prosumer\#2 turned off, switch it on during the experiment and turn it off once again towards the end.

In Fig. 12, we see the results logged on the operator GUI. Initially, the entire PV power is being exported to the grid. The exported power is slightly below the PV due to the losses in the LV grid. When Prosumer\#2 is turned on, it starts consuming the PV generation and the grid export starts to drop. As the power control loop is active the dynamic load exactly matches the PV generation and the grid export has dropped to zero. The transition takes approximately $40 \mathrm{~ms}$ or two cycles, which is again consistent with the results from Fig. 11. The same behaviour takes place is reverse when the Prosumer\#2 is then turned off.

\section{CONCLusion}

We identified a gap in the distributed PHIL control literature, where all the relevant measurements are not directly wired to one controller. In the real world, an increasing number of prosumers on the LV grid may not have direct voltage measurements from PCC due to distance limits. We 


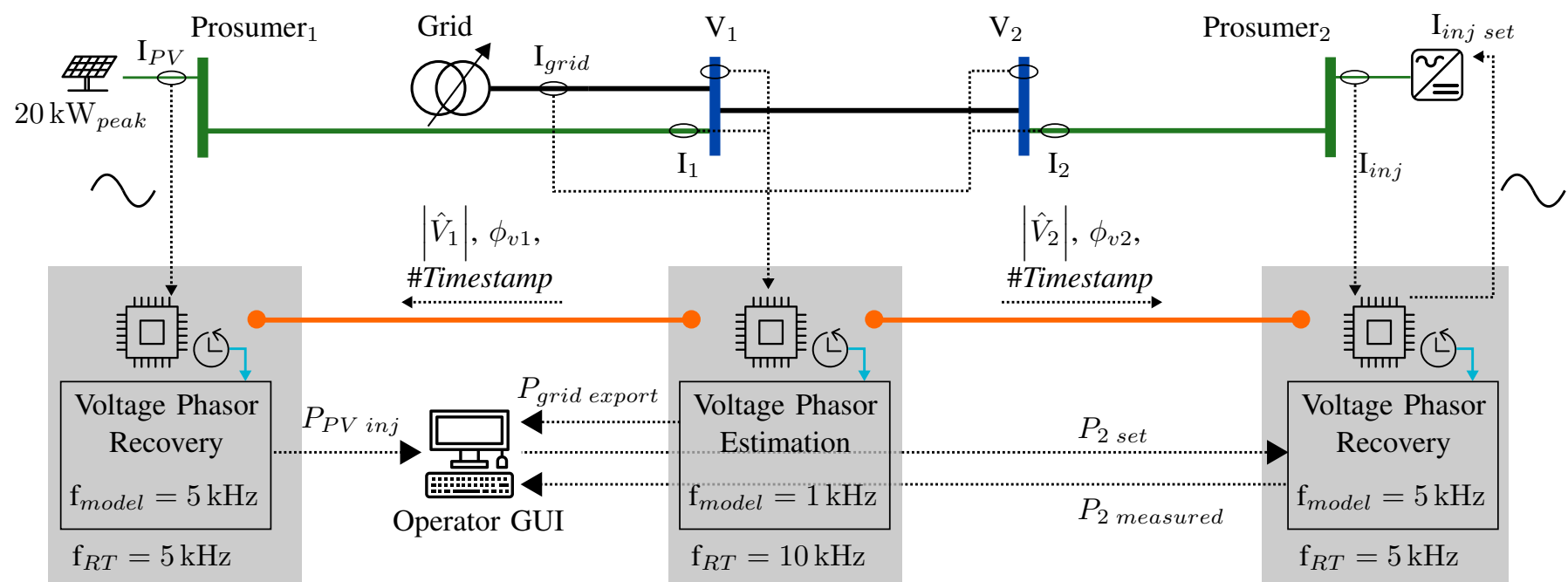

Prosumer controller \#1

Grid controller

Prosumer controller \#2

Fig. 11. Schematic of the PHIL experiment for matching the PV production as a dynamic load to make net export zero in grid connected mode. Prosumer\#1 is connected to $20 \mathrm{~kW}_{\text {peak }} \mathrm{PV}$ and Prosumer\#2 is programmed as a dynamic load with a power setpoint. Grid controller measures and analyses the voltages.
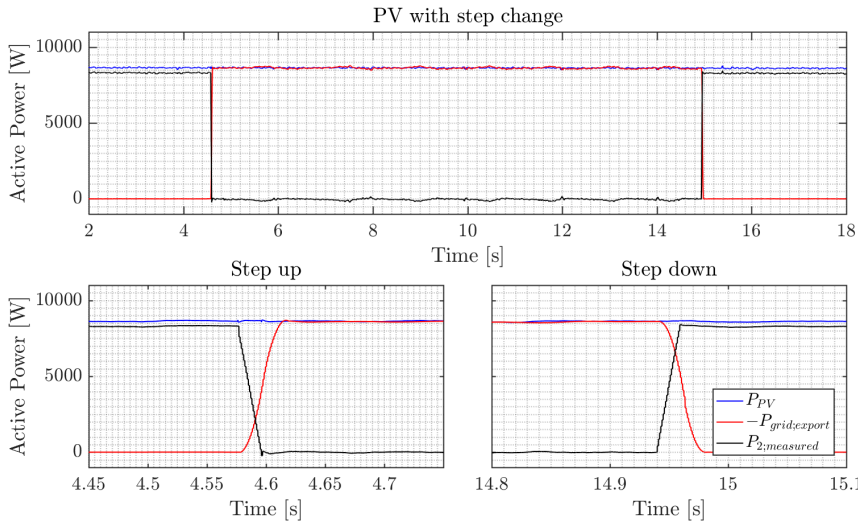

Fig. 12. Grid export with Prosumer\#2 matching the PV Power

propose a frequency-shifting and filtering based fundamental signal analysis and recovery method between RT controllers connected to an unifying clock source, such as GPS. This method can work with variable delay between the sending and receiving end. We validated the proposed algorithm on a PHIL test-bed and then showed that it can be readily used in a variety of PHIL experiments in islanded and grid connected mode. The tool is now being used in the CoSES lab for all PHIL experiments.

\section{REFERENCES}

[1] V. S. Perić, T. Hamacher, A. Mohapatra, F. Christiange, D. Zinsmeister, P. Tzscheutschler, and C. Aigner, "CoSES Laboratory for Combined Energy Systems At TU Munich," IEEE Power and Energy Society General Meeting.

[2] "IEEE standard definitions for the measurement of electric power quantities under sinusoidal, nonsinusoidal, balanced, or unbalanced conditions," IEEE Std 1459-2010, pp. 1-50, 2010.

[3] J. W. Cooley and J. W. Tukey, "An algorithm for the machine calculation of complex fourier series," Mathematics of computation, vol. 19, no. 90, pp. 297-301, 1965.

[4] J. Barros, R. I. Diego, and M. de Apraiz, "Applications of wavelet transform for analysis of harmonic distortion in power systems: A re- view," IEEE Transactions on Instrumentation and Measurement, vol. 61 pp. 2604-2611, Oct 2012.

[5] P. Rodriguez, A. Luna, I. Etxeberria, J. R. Hermoso, and R. Teodorescu, "Multiple second order generalized integrators for harmonic synchronization of power converters," in 2009 IEEE Energy Conversion Congress and Exposition, pp. 2239-2246, Sep. 2009.

[6] A. A. Girgis, W. B. Chang, and E. B. Makram, "A digital recursive measurement scheme for online tracking of power system harmonics," IEEE Transactions on Power Delivery, vol. 6, pp. 1153-1160, July 1991.

[7] Z. Shuai, J. Zhang, L. Tang, Z. Teng, and H. Wen, "Frequency shifting and filtering algorithm for power system harmonic estimation," IEEE Transactions on Industrial Informatics, vol. 15, no. 3, pp. 1554-1565, 2019.

[8] S. Tnani, M. Mazaudier, A. Berthon, and S. Diop, "Comparison between different real-time harmonic analysis methods for control of electrical machines," 1994.

[9] A. E. Kibar and O. Salor, "Harmonics analysis of power signals using quadrature amplitude modulation," in 2016 24th Signal Processing and Communication Application Conference (SIU), pp. 1937-1940, May 2016.

[10] E. Sezgin and O. Salor, "Analysis of power system harmonic subgroups of the electric arc furnace currents based on a hybrid time-frequency analysis method," IEEE Transactions on Industry Applications, pp. 1-1, 2019.

[11] E. Sezgin, Development of new frequency and time-frequency analysis approaches on power system signals and their implementation on hardware-in-the-loop framework. PhD thesis, Gazi University, Ankara,Turkey, 72020.

[12] R. G. Lyons, Understanding digital signal processing, 3/E. Pearson Education India, 2004.

[13] K. Duda, "Accurate, guaranteed stable, sliding discrete fourier transform [dsp tips \& tricks]," IEEE Signal Processing Magazine, vol. 27, no. 6, pp. $124-127,2010$.

[14] P. Romano and M. Paolone, "An enhanced interpolated-modulated sliding dft for high reporting rate pmus," in 2014 IEEE International Workshop on Applied Measurements for Power Systems Proceedings (AMPS), pp. 1-6, 2014.

[15] C. M. Orallo, I. Carugati, S. Maestri, P. G. Donato, D. Carrica, and M. Benedetti, "Harmonics measurement with a modulated sliding discrete fourier transform algorithm," IEEE Transactions on Instrumentation and Measurement, vol. 63, no. 4, pp. 781-793, 2014.

[16] "Egston power product portfolio." https://www.egstonpower.com Accessed: 03-09-2021.

[17] "National Instruments." https://www.ni.com Accessed: 03-09-2021.

[18] “LEM Europe GmbH.” https://www.lem.com Accessed: 03-09-2021. 